Dietary Chinese Herbs 
Yanze Liu - Zhimin Wang

Junzeng Zhang

Editors

\section{Dietary Chinese Herbs}

Chemistry, Pharmacology

and Clinical Evidence

Springer 


\section{Editors}

Yanze Liu

Institute of Medicinal Plant Development

Chinese Academy of Medical Sciences

and Peking Union Medical College

Beijing

China

\section{Zhimin Wang}

Institute of Chinese Materia Medica

China Academy of Chinese Medical

Sciences

Beijing

China
Junzeng Zhang

Aquatic and Crop Resource Development

National Research Council of Canada

Halifax

Canada

ISBN 978-3-211-99447-4

ISBN 978-3-211-99448-1 (eBook)

DOI 10.1007/978-3-211-99448-1

Library of Congress Control Number: 2015934051

Springer Wien Heidelberg New York Dordrecht London

(C) Springer-Verlag Wien 2015

This work is subject to copyright. All rights are reserved by the Publisher, whether the whole or part of the material is concerned, specifically the rights of translation, reprinting, reuse of illustrations, recitation, broadcasting, reproduction on microfilms or in any other physical way, and transmission or information storage and retrieval, electronic adaptation, computer software, or by similar or dissimilar methodology now known or hereafter developed.

The use of general descriptive names, registered names, trademarks, service marks, etc. in this publication does not imply, even in the absence of a specific statement, that such names are exempt from the relevant protective laws and regulations and therefore free for general use.

The publisher, the authors and the editors are safe to assume that the advice and information in this book are believed to be true and accurate at the date of publication. Neither the publisher nor the authors or the editors give a warranty, express or implied, with respect to the material contained herein or for any errors or omissions that may have been made.

Printed on acid-free paper

Springer-Verlag GmbH Wien is part of Springer Science+Business Media (www.springer.com) 


\section{Foreword I}

"We are what we eat." This old saying rings true as an increasingly large body of scientific evidence has revealed the close relationship between food and health. In China, we say "disease comes through the mouth," meaning that bad food choice leads to illness. Indeed, human life relies on three basic resources: the air we breathe, the water we drink, and the food we eat. Human health is thus the outcome of constant interplay among genetic background, environmental condition, and food choice.

In traditional Chinese medicine (TCM), illness means imbalance and the focus is always to adjust and restore the balance. Over 2,000 years, TCM has used herbs and other approaches such as acupuncture, for treatment and prevention of diseases. The prevention approach or health conservation has been an important part of TCM. The oldest herbal "Shen Nong Ben Cao Jing" (Shen Nong Materia Medica) has 120 nontoxic herbs categorized as a superior group, and most of them are tonics and used for health preservation. This forms the basis of using medicated foods and dietary herbs in health maintenance - "food is medicine."

This book, "Dietary Chinese Herbs: Chemistry, Pharmacology and Clinical Evidence," edited by Drs. Liu, Wang, and Zhang brings to readers concise reviews of the history of dietary herbs in China, the perspective of natural health products and nutraceutical application relevant to dietary Chinese herbs and ingredients, and focuses on 86 selected herbs that are commonly used and regulated as food or heath food raw materials in China. The editors are well-established researchers, all with background in traditional Chinese medicine and phytochemistry, natural products chemistry, or medicinal chemistry. My colleague Dr. Liu had worked on Chinese herbal research in leading institutions in the United States for many years, while Dr. Wang is an expert in China on TCM quality and standardization, a member of the Chinese Pharmacopoeia Commission. Dr. Zhang currently leads the functional ingredients chemistry R\&D of a national program on natural health products and functional foods in Canada. They are all passionate about the health benefits of dietary herbs, the bioactive components, mechanisms of actions, and new health food products development. 
I have been working in the field of medicinal plant research for more than 60 years, but the love and understanding for medicinal plants never ceases. I am glad to see the book Dietary Chinese Herbs edited by Drs. Liu, Wang, and Zhang. Among the books that have touched on TCM for its dietary application, this one is unique as it provides a collection of high-level scientific literature reviews on the most commonly used dietary Chinese herbs. It will be a good reference book for researchers, graduate students, and R\&D managers from industry of natural health products, dietary supplements, and functional foods.

Peigen Xiao

Academician, Chinese Academy of Engineering and Honorary Director, Institute of Medicinal Plant Development Chinese Academy of Medical Sciences and Peking Union Medical College

Beijing, China 


\section{Foreword II}

Traditional Chinese medicine (TCM) and Chinese materia medica (CMM) have thousands of years of history and are important elements of Chinese culture. Most CMM are derived from botanical materials or plants, so they are called Chinese herbal medicines. Historically, decoctions and herbal teas have been the most popular and effective forms; however, tablets, pills, capsules, lozenges, and injections have become the mainstream of modern CMM. Throughout the history of clinical practice, people have recognized and recorded properties such as taste, function, therapeutic effect, dosage, administration, side effects, and toxicity of various herbs. People also understand that some herbs are mainly used for therapeutic purpose, while others are used for their health-maintaining properties. Also, some herbs are mainly consumed as foods, although they show certain biological function and health benefits.

Drs. Liu, Wang, and Zhang have each been working on CMM and natural health products for more than 30 years. With similar research experience and interests, they selected 86 herbs with health-maintaining properties and invited a group of experienced researchers from China, USA, and Canada who worked in this area to contribute to the book shown here.

The book is composed of 88 chapters, including two introductory chapters and 86 chapters on specific dietary herbs, such as renshen (Panax ginseng), danggui (Angelica sinensis), shanyao (Dioscorea opposita), bajitian (Morinda officinalis), gegen (Pueraria lobata), baiguo (Ginkgo biloba), gouqi (Lycium barbarum), luhui (Aloe barbadensis), jinyinhua (Lonicera japonica), juju (Cichorium glandulosum), lingzhi (Ganoderma lucidum), and yangqicai (Sargassum fusiforme). Each herb is described based on botanical identity, chemical constituents, pharmacological studies, TCM application and dietary usage, clinical evidence, safety evaluation and toxicity data. In the botanical identity section, color photos of plants showing typical plant morphology give readers clear information on the sources. Main components, bioactive compounds, and marker compounds with updated references are included in the chemical constituents section. In the pharmacological studies section, traditional, confirmed, and newly discovered pharmacological activities are summarized. In the TCM application and dietary usage section, examples of dietary 
usage are included. As one of the current foci on safety and toxicity, authors also included relevant data published for readers' reference.

I have been working in this area, especially pharmacological research, for more than three decades and have traveled to Japan, USA, Korea, Malaysia, and other countries for research and academic exchanges on herbal medicine. It is my honor to have this opportunity to introduce the book. I am sure that readers who are interested in herbal medicine can find what they are looking for.

Xiaobo Sun

Professor and Director

Institute of Medicinal Plant Development

Chinese Academy of Medical Sciences and Peking Union Medical College

Beijing, China 


\section{Preface}

The concept of "food is medicine" can be dated back to 2,000 years ago in the earliest traditional Chinese medicine (TCM) literature "Huang Di Nei Jing” (“黄帝 内经”, or “Emperor's Inner Canon," 475 BCE-220 CE), where it emphasized the importance of maintenance or preservation of wellness and health and the prevention of illness and diseases, with the old but still valid notion "the best doctor prevents, not treats illness." As such, maintaining system balance with the use of food, herbal medicine, and other complementary approaches in an integrated manner is the essence of TCM for disease prevention and treatment.

Over thousands of years, food materials have been continuously studied for their health benefits, while a wide range of TCM herbs have also been investigated and incorporated into the daily diet for maintaining general wellness or prevention of certain diseases in China. In the West, the convergence of food and medicine driven by market force has led to increasing demand for dietary supplements, natural health products, nutraceuticals, or functional foods. This trend has also stimulated interest in the West to look at many natural materials that could be used as sources for developing new, effective, and safe ingredients to capture the rapidly expanding opportunity in the global market place.

The book idea came out a few years ago when the three of us, working in China, Canada, and the United States at that time, were all involved in studying or reviewing the bioactive components of dietary herbs. We realized that, although there is a large and rapidly growing body of scientific information in the literature for various Chinese herbs, it is somewhat scattered and not specific toward dietary applications. The book Dietary Chinese Herbs is our first attempt to bring together selected TCM herbs and highlight the plant source, traditional use, main chemical components, biological and pharmacological activities, and clinical and dietary uses. It is not meant to cover all the available information, but rather to introduce these selected herbs with some of the research findings and relevant information on TCM and dietary uses in China. We hope it can be a useful reference for researchers and students in academia, R\&D, and business managers in dietary supplement, natural health products, and the functional food industry. 
The contents are arranged by starting with a brief chronological review of Chinese literatures on dietary herbs, overview of food and nutraceutical applications, and followed by chapters dedicated to each selected dietary herb. For each dietary herb or group of similar herbs, the plant source, processing method, TCM, and dietary uses will be introduced, and then followed by up-to-date literature reviews of some key chemical, pharmacological, and clinical studies.

In the preparation of this book, we are grateful to the dedication of all contributors for their rich knowledge and diverse perspectives in organizing the chapter contents. We also appreciate the time and efforts of the following students from the Applied Human Nutrition program, Mount Saint Vincent University, Halifax, Canada for language editing assistance: Laura Bellussi, Elizabeth Dickson, Shelby MacGregor, Esther Adsett, Kennedy Bennicke, Gillian Blundon, Ashleigh Cassell, Sarah Creelman, Hayley Ewing, Susan Gillespie, Michelle Higgins, Liza Hooper, Tika Jakobsen, Joseph Legere, Molly McLaughlin, Megan Phillips, Katrina Ross, Katie Tanner, Amanda Worth, Erada Alghamdi, Kim Allen, Melissa Church, Angela Crouquet, Virginia De Silva, Sarah Hallett, Mallory Harvie, Katie Inkpen, Kristen Lutes, Sarah McKay, Janie Nelson-Isenor, Olivia Newton, Leila Shaw, Clarissa Smith, and Mylene Whynot.

We are also greatly indebted to Qiwei Zhang for his help in coordination of the manuscripts and assistance in editing, as well as to Bohdan L. Luhovyy and Phillip Joy for organizing the English editing work.

Last but not least, we would like to thank the publisher Springer and its publishing editors Stephen Soehlen and Annelies Kersbergen for all the patience and support over the years to bring this from an idea to reality.

Yanze Liu Zhimin Wang Junzeng Zhang 


\section{Contents}

\section{Part I Introduction}

1 A Brief History of Dietary Chinese Herbs . . . . . . . . . . . . 3 Junzeng Zhang, Zhimin Wang and Yanze Liu

2 Food and Nutraceutical Applications of Chinese Herbal Products . . . . . . . . . . . . . . . . . . . . . . . . . . . 23

Priya Kathirvel, Phillip Joy and Bohdan L. Luhovyy

Part II Root, Rhizome, Tuber, and Bulb Materials

3 Achyranthes bidentata $\mathrm{Bl}$. 牛膝 (Niuxi, Twotooth Achyranthes Root) . . . . . . . . . . . . . . . . . 45 Minhui Li

4 Alisma orientalis (Sam.) Juzep. 泽泻 (Zexie, Alismatis Rhizoma) . . . . . . . . . . . . . . 53 Min Fu and Ling Wang

$5 \quad$ Alpinia officinarum Hance 高良姜 (Gaoliangjiang, galangal) . . . . . 61 Ping Ding

6 Angelica dahurica (Fish. ex Hoffm.) Benth. et Hook. f. 白芷 (Baizhi, Chinese Angelica) . . . . . . . . . . . . . . . . . 69 Minhui Li

7 Angelica sinensis (Oliv.) Diels 当归 (Danggui, Dongkuai) . . . . . . 75 Jun Xu, Hubiao Chen and Quanbin Han 
8 Asparagus cochinchinensis (Lour.) Merr. 天冬

(Tiandong, Chinese Asparagus)

Feng Zhang, Yin Lu, Wenhui Qian and Zifan Pei

9 Astragalus membranaceus 黄芪 (Huangqi, Milkvetch Root) Hua Wei

10 Codonopsis pilosula 党参 (Dangshen, Pilose Asiabell) . . . . . . . . En-yuan Zhu

11 Curcuma longa L. 姜黄 (Jianghuang, Common Turmeric) . . . . . 107 Jing-jing Zhu

12 Dioscorea opposite Thunb. 山药 (Shanyao, Chinese Yam) . . . . . . . 113 Sue-Joan Chang, Chun-Yung Huang and Yin-Ching Chan

13 Gastrodia elata Blume. 天麻 (Tianma, Gastrodia Tuber) . . . . . . . 127 Hui-Min Gao

14 Glycyrrhiza uralensis 甘草 (Gancao, Licorice). . . . . . . . . . . . 135 Sue-Joan Chang, Yin-Ching Chan and Wen-Jen Yu

15 Lilium lancifolium 百合 (Baihe, Tiger Lily) . . . . . . . . . . . . . 147 Yanze Liu

16 Morinda officinalis How 巴戟天 (Bajitian) . . . . . . . . . . . . . 153 Ping Ding

17 Ophiopogon japonicus (Thunb.) Ker-Gawl. 麦冬 (Maidong, Fountain Plant) . . . . . . . . . . . . . . . . . . . . . 161 Li-mei Lin and Xiao-liang Zhao

18 Paeonia lactiflora Pall. 苟药 (Shaoyao, Chinese Herbaceous Peony)

Feng Zhang, Yin Lu, Wenhui Qian and Zifan Pei

19 Panax ginseng 人参 (Renshen, Ginseng) . . . . . . . . . . . . . . 175 Yuqing Zhao

20 Panax notoginseng (Burk.) F.H. Chen 三七 (Sanqi, Notoginseng)

Yuqing Zhao 
21 Panax quinquefolius L. 西洋参 (Xiyangshen, American Ginseng).

Yuqing Zhao

22 Platycodon grandiflorum (Jacq.) A. DC. 桔梗

(Jiegeng, Balloonflower) . . . . . . . . . . . . . . . . . . . . . 205

Muxin Gong and Xuran Lu

23 Polygonatum cyrtonema Hua 黄精 (Huangjing). . . . . . . . . . 213 Ta-si Liu and Bei Xu

24 Polygonatum odoratum (Mill.) Druce 玉竹 (Yuzhu) . . . . . . . . . . 219 Ta-si Liu and Ying-Jiao Liu

25 Polygonum multiflorum Thunb. 何首乌

(Heshouwu, Tuber Fleeceflower Root) . . . . . . . . . . . . . . . . . . 227

Raorao Li and Hui-Min Gao

26 Pueraria lobata (Willd.) Ohwi 葛根 (Gegen, Kudzu) . . . . . . . . . . 235 Minhui Li

27 Rehmannia glutinosa Libosch. 地黄 (Dihuang, Rehmannia). . . . . 247 Pengfei Li and Mingsan Miao

28 Rhodiola crenulata L. 红景天 (Hongjingtian, Red-Spotted Stonecrops) . . . . . . . . . . . . . . . . . . . . 255

Tao Guo

29 Salvia miltiorrhiza Bonge 丹参 (Danshen, Red Sage) . . . . . . . . . 265 Yanze Liu

30 Zingiber officinale (Willd.) Rosc. 姜 (Jiang, Common Ginger) . . . . 273 Hui-Min Gao

\section{Part III Fruit or Seed Materials}

31 Alpinia oxyphylla Miquel 㿽智仁 (Yizhi Ren, Sharpleaf Galangal)

Lihong $\mathrm{Wu}$

32 Amomum villosum 砂仁 (Sharen, Amomum Fruit) Li-hua $\mathrm{Gu}$ 
33 Arctium lappa L. 牛蒡子 (Niubangzi, Great Burdock). . . . . . . . 301 Yang Zhao and Xin Zhou

34 Canarium album (Lour.) Raeusch. 青果 (Qingguo,

Chinese Olive $\ldots \ldots \ldots$. . . . . . . . . . . . . . . . . . 307

Chunnian $\mathrm{He}$

35 Cassia Obtusifolia L. 决明子 (Juemingzi, Semen Cassiae) . . . . . . 315 Yulan Wang

36 Chaenomeles speciosa 木瓜 (Mugua, Flowering Quince) . . . . . . 321 Caifang Wang

37 Citrus medica L. var. sarcodactylis Swingle 佛手 (Foshou, Finger Citron) . . . . . . . . . . . . . . . . . . . . . . 327

Qi-wei Zhang

38 Citrus reticulata Blanco and Cultivars 橘皮 (Jupi, Mandarin Orange Peel) . . . . . . . . . . . . . . . 333 Qi-wei Zhang

39 Coix lacryma-jobi L. var. ma-yuen (Roman.) Stapf 薏苡仁 (Yiyiren, Jobstears) . . . . . . . . . . . . . . . . . . . . . . . . 339 Fei Yu, Yazhuo Li, Jun Zhang and Changxiao Liu

40 Cornus officinalis Sieb. et Zucc. 山荣获 (Shanzhuyu, Medicinal Dogwood) . . . . . . . . . . . . . . . . . . . . . . . . . . . . 347 Jin Yang

41 Crataegus pinnatifida Bge. 山楂 (Shanzha, Hawthorn Fruit) . . . . 355 Caifang Wang

42 Dimocarpus longan Lour. 龙眼肉 (Longyanrou, Longan) . . . . . . 363 Yang Yi and Ming-wei Zhang

43 Euryale ferox 芡实 (Qianshi, Gordon Euryale Seed) . . . . . . . . . . 371 Caifang Wang

44 Gardenia jasminoides Ellis 柧子 (Zhizi, Capejasmine) . . . . . . . . . 379 Jianhui Liu and Fei Yin

45 Ginkgo biloba L. 银杏 (Yinxing, Baiguo, Ginkgo) . . . . . . . . . . 391 Yingqin $\mathrm{Li}$ and Chun $\mathrm{Hu}$ 
46 Hippophae rhamnoides L. 沙棘 (Shaji, Common

Sea-buckthorn). . . . . . . . . . . . . . . . . . . 403

Yingqin $\mathrm{Li}$ and Chun $\mathrm{Hu}$

47 Hovenia dulcis Thunb. 枳椇子 (Zhijuzi, Oriental

Raisin Tree Seed) . . . . . . . . . . . . . . . . . . . . . . . . . . 417

Tongxiang Liu, Shengyu Hua and Zongwei Wang

48 Lycium barbarum L. 枸杞子 (Gouqizi, Wolfberry) . . . . . . . . . 4 425 Jin Yang

49 Siraitia grosvenorii Swingle 罗汉果 (Luo Han Guo) . . . . . . . . . 431 Chun Li

50 Myristica fragrans Houtt. 肉豆莞 (Roudoukou, Nutmeg) . . . . . . . 439 Ping Ding

51 Phyllanthus emblica L. 余甘子 (Yuganzi, Indian Gooseberry) . . . . 447 Yanze Liu and Fan Liu

52 Piper nigrum L. 黑胡椒 (Heihujiao, Black Pepper) . . . . . . . . . 457 Jianhui Liu and Fei Yin

53 Prunella vulgaris L. 夏枯草 (Xiakucao, Common Selfheal) . . . . . 469 Li-mei Lin, Hui-Min Gao and Jing-jing Zhu

54 Prunus armeniaca L. 苦杏仁 (Kuxingren, Apricot) . . . . . . . . . . 477 Feng Zhang, Yin Lu, Wenhui Qian and Zifan Pei

55 Prunus mume (Sieb.) Sieb. et Zucc. 乌梅 (Wumei, Japanese Apricot).

Jianhui Liu and Fei Yin

56 Rosa davurica Pall 刺玫果 (Cimeiguo, Dahurian Rose Fruit). . . . . 495 Min Fu and Yanze Liu

57 Rosa laevigata Michx. 金樱子 (Jinyingzi, Cherokee Rose) . . . . . . . 501 Xiaozhe Zhang

58 Rubus chingii 覆盆子 (Fupenzi, Immature Raspberry Fruit) . . . . 509 Tongxiang Liu, Shengyu Hua and Zongwei Wang

59 Schisandra chinensis 五味子 (Wuweizi, Chinese Magnoliavine) . . . 519 Jing-jing Zhu 
60 Sesamum indicum L. 黑芝麻 (Heizhima, Black Sesame) . . . . . . 525 Haixia Li and Chunbo Lu

61 Sterculia lychnophora Hance 㭌大海 (Pangdahai, Malva Nut Tree $) \ldots \ldots \ldots \ldots \ldots \ldots 55$ Chun Li

62 Terminalia chebula Retz. 诃子 (Hezi, Chebulic Myrobalan). . . . . 543 Chunnian $\mathrm{He}$

63 Vigna umbellata (Thunb.) Ohwi et Ohashi or Vigna angularis (Willd.) Ohwi et Ohashi 赤小豆 (Chixiaodou, Rice Bean) . . . . . . 551 Yingfang Wei, Jie Yan, Fei Long and Guanghua Lu

64 Ziziphus jujuba Mill. 大東 (Dazao, Common Jujube) . . . . . . . . 561 Panbo Qiu and Mingsan Miao

65 Ziziphus jujuba var. spinosa 酸菄仁 (Suanzaoren). . . . . . . . . . 569 Panbo Qiu and Mingsan Miao

Part IV Aerial Part, Stem, Stem Bark, and Leaf Materials

66 Aloe barbadensis Miller 芦荟 (Luhui, Aloe vera). . . . . . . . . . . . 577 Muxin Gong and Xuran Lu

67 Cinnamomum cassia Presl. 肉桂 (Rougui, Cassia Bark Tree) . . . . 587 Tingting Feng, Xiongli Liu, Bing Lin and Ying Zhou

68 Dendrobium nobile Lindl. 石斛 (Shihu, Dendrobium) . . . . . . . . 597 Hong $\mathrm{Xu}$ and Zhengtao Wang

69 Epimedium brevicornu Maxim. 淫羊藿 (Yinyanghuo, Barrenwort) . . . . . . . . . . . . . . . . . . . . . 605 Li-hua Yan

70 Gynostemma pentaphyllum (Thunb.) Makino 绞股蓝 (Jiaogulan, Fiveleaf Gynostemma) . . . . . . . . . . . . . . 615 Li-hua Yan

71 Houttuynia cordata Thunb 鱼腥草 (Yuxingcao, Houttuynia) . . . . 623 Qi-wei Zhang 
72 Mentha haplocalyx Briq. 薄荷 (Bohe, Mint) . . . . . . . . . . . 631 Feng Zhang, Yin Lu, Wenhui Qian and Zifan Pei

73 Mosla chinensis Maxim. 香薷 (Xiangru, Chinese Mosla Herb) . . 637 Zhimin Wang

74 Portulaca oleracea L. 马齿苋 (Machixian, Purslane) . . . . . . . . . . 645 Raorao Li and Hui-Min Gao

75 Taraxacum mongolicum 蒲公英 (Pugongying, Dandelion) . . . . . . 651 Chun $\mathrm{Hu}$

Part V Flower or Flower Bud Materials

76 Carthamus tinctorius L. 红花 (Honghua, Safflower). . . . . . . . . 671 Zhuju Wang and Xidan Zhou

77 Chrysanthemum morifolium Ramat 菊花 (Juhua, Florists Chrysanthemum) . . . . . . . . . . . . . . . . . . 681 Chun $\mathrm{Hu}$

78 Lonicera japonica Thunb 金银花 (Jinyinhua, Honey Suckle) . . . . . 693 Haixia $\mathrm{Li}$ and Chunbo Lu

79 Sophora japonica L. 槐花 (Huaihua, Japanese Pagodatree Flower Bud) 703 Raorao Li and Hui-Min Gao

\section{Part VI Multiple-part Materials}

80 Cichorium glandulosum Bioss. Et Huet 菊苣 (Juju, Chicory). . . . 711 Haji Akber Aisa and Xuelei Xin

81 Morus alba L. 桑 $($ Sang, White Mulberry) . . . . . . . . . . . . 721 Hua Wei

82 Nelumbo nucifera Gaertn. 荷 (He, Lotus) Xiao-liang Zhao

83 Perilla frutescens (L.) Britt. 紫苏 (Zisu, Common Perilla and Purple Common Perilla). 
84 Plantago asiatica L. 车前 (Cheqian, Asiatic Plantain) . . . . . . . . . 749 Li Yang

Part VII Fungi, Marine Algae, and Other Materials

85 Ganoderma lucidum 灵芝 (Lingzhi, Ganoderma) . . . . . . . . . . 759

Caixia Dong and Quanbin Han

86 Laminaria japonica Aresch. and Ecklonia Kurome

Okam. 昆布 (Kunbu, Kelp) . . . . . . . . . . . . . . . . . . . . . . 767

Xiaoliang Zhao, Guangling Jiao, Jiandong Wu,

Junzeng Zhang and Guangli Yu

87 Poria cocos (Schw.) Wolf 获苓 (Fuling, Indian Bread) . . . . . . . . 781

Xiao-jun Gou, Gang He and Xiao-qiang Guo

88 Sargassum fusiforme (Harv.) Setch. 羊栖荣 (Yangqicai, Hijiki) . . . 789 Yanze Liu

Latin Index . . . . . . . . . . . . . . . . . . . . . . . . . . . . . . 797 


\section{Editors and Contributors}

\section{About the Editors}

Dr. Yanze Liu graduated from Henan University of Traditional Chinese Medicine (HUTCM) and received his Ph.D. in Organic Chemistry from Zhengzhou University (ZZU), China. He was a visiting scholar at the Institute of Materia Medica, Chinese Academy of Medical Science (CAMC 1985), Beijing, and Okayama University, Japan (1988-1992), who focused on the isolation and structure determination of botanical tannins and polyphenols. He was promoted to associate professor (1991) and full professor (1995), and served as associate director and director in the Department of Organic and Natural Product Chemistry since 1992 at HUTCM. In 1999 Dr. Liu went to the Department of Chemistry, The Pennsylvania State University for his visiting research, and then was transferred to Bio-organic and Natural Product Lab, McLean Hospital/Harvard Medical School, working as postdoc fellow and then as instructor.

Since 2010, Dr. Liu was invited to take the position in charge of Chinese Herbal Medicines, a newly founded journal published in English as a senior editor and serve as a full professor in Beijing Union Medical College/CAMC. Dr. Liu as a principal investigator took charge of three national projects of National Natural Science Foundation of China (NSFC) since 1991, one Key Project of National Scientific Research of 9th Five-Year Plan, and one Project of Henan Provincial Foundation for Excellent Young Scientist.

Dr. Liu is a guest speaker of New England School of Acupuncture, Boston, and adjunct professor of ZZU, Beifang University of Nationality, Harbin University of Commerce, and Nanyang Medical College. He was a member of experts of National Toxicology Program under NIH to evaluate 12th Report on Carcinogens. Dr. Liu received a dozen awards and honorary titles including Excellent Young Scientist of Henan Province given by Henan provincial government; published more than 120 papers on national and international journals; invented and patented flash extractor and concentrator for herbal medicine research; and licensed and patented a number of new herbal health products. 
Dr. Liu's research was highlighted in the Progress Report of NSFC (1996) as the only one in the area of chemistry and also in the cover of Chinese Herbal Medicines (April, 2014). His research interests include natural product chemistry, R\&D of healthy food and herbal products, quality control and standardization of herbal products, analysis and characterization of tannin and polyphenol, and crystallization of organic compounds.

Dr. Zhimin Wang is the Chief Professor of China Academy of Chinese Medical Sciences (CACMS), Director of National Engineering Laboratory for Quality Control Technology of Chinese Herbal Medicine, and Deputy Director of State Key Laboratory for New Technology in Manufacture Process of Chinese Medicines, from Institute of Chinese Materia Medica (ICMM), CACMS. Dr. Wang obtained his B.Sc. (1984) in Pharmacy from Henan University of Traditional Chinese Medicine, M.Sc. (1991) in Phytochemistry from China Pharmaceutical University, and Ph.D. (1994) in Medicinal Chemistry from Institute of Materia Medica, Chinese Academy of Medical Sciences and Peking Union Medical College, China.

Prof. Wang mainly engages in phytochemistry, quality standard, and quality evaluation of Chinese herbal medicines, and antitumor new drug development. As a principal investigator, he was involved in over 55 research projects related to basic research (National 973 Project) and R\&D of new medicines and quality control of Chinese medicines since he joined ICMM. Dr. Wang received over fifteen scientific awards, published more than 300 research papers, and 16 books, including four books in which he served as editor-in-chief. As a senior expert for authoritative evaluation of novel food in China, he is responsible for reviewing the chemistry, quality, and production process of novel food from botanical resources or folk medicines.

Dr. Junzeng Zhang is a research officer from the Aquatic and Crop Resource Development, National Research Council of Canada (NRC). He is the current board member of the Canadian Institute of Chinese Medicinal Research (CICMR) and the Natural Health Products Research Society of Canada (NHPRS). Dr. Zhang obtained his B.Sc. (1984) in Pharmacy from Henan University of Traditional Chinese Medicine, and M.Sc. (1991) and Ph.D. (1994) in natural products chemistry from Institute of Materia Medica, Chinese Academy of Medical Sciences and Peking Union Medical College, China. He then did his postdoctoral research in Peking University, China; Rutgers, the State University of New Jersey, USA, and INRSInstitut Armand-Frappier, Canada, on natural products-based drug discovery and nutraceutical ingredients characterization.

Dr. Zhang then joined Ocean Nutrition Canada Ltd. (ONC, now a division of DSM) as a senior research scientist in the year 2000 to work on a marine-based natural health products and functional food discovery and development, later assumed the role of group leader and principal research scientist in natural products chemistry, and then the manager of licensing and research collaborations at ONC by focusing on potential product licensing opportunities and coordinating R\&D collaborations in 2005. While working at ONC, he also took a part-time program at 
the Sobey School of Business, Saint Mary's University, and received his MBA in 2006.

Dr. Zhang joined the National Research Council of Canada as a research officer in April 2006, at the Institute for Nutrisciences and Health and now the Aquatic and Crop Resource Development portfolio, the Division of Life Sciences at NRC. He is currently a pillar lead for NRC's Natural Health Products and Functional Ingredients (NHP/FI) program. His research expertise includes natural products-based drug/nutraceutical discovery from bioresources, including dietary Chinese herbs; microbial or enzymatic transformation of natural products; in vitro and in vivo metabolism of bioactive natural products; and the application of metabolomics tools in natural health products research and development, including quality assessment.

\section{Contributors}

Haji Akber Aisa Xinjiang Technical Institute of Physics and Chemistry, Chinese Academy of Sciences, Urumqi, Xinjiang, China

Yin-Ching Chan Department of Food and Nutrition, Providence University, Taichung, Taiwan

Sue-Joan Chang Department of Life Sciences, National Cheng Kung University, Tainan, Taiwan

Hubiao Chen School of Chinese Medicine, Hong Kong Baptist University, Hong Kong, China

Ping Ding School of Chinese Materia Medica, Guangzhou University of Chinese Medicine, Guangzhou, China

Caixia Dong School of Chinese Medicine, Hong Kong Baptist University, Kowloon, Hong Kong

Tingting Feng College of Pharmacy, Guizhou University, Guiyang, Guizhou, China; Guizhou Engineering Center for Innovative Traditional Chinese Medicine and Ethnic Medicine, Guiyang, Guizhou, China

Min Fu McGill University, Montreal, QC, Canada

Hui-Min Gao Institute of Chinese Materia Medica, China Academy of Chinese Medical Sciences, Beijing, China

Muxin Gong School of Traditional Chinese Medicine, Capital Medical University, Beijing, China

Xiao-jun Gou Key Laboratory of Medicinal and Edible Plants Resources Development of Sichuan Education Department, Chengdu University, Chengdu, Sichuan, China 
Li-hua Gu Shanghai University of Traditional Chinese Medicine, Shanghai, China

Tao Guo School of Life and Engineering, Lanzhou University of Technology, Lanzhou, China

Xiao-qiang Guo Key Laboratory of Medicinal and Edible Plants Resources Development of Sichuan Education Department, Chengdu University, Chengdu, Sichuan, China

Quanbin Han School of Chinese Medicine, Hong Kong Baptist University, Hong Kong, China

Chunnian He Institute of Medicinal Plant Development, Chinese Academy of Medical Science, Beijing, China

Gang He Key Laboratory of Medicinal and Edible Plants Resources Development of Sichuan Education Department, Chengdu University, Chengdu, Sichuan, China

Chun Hu Nutrilite Health Institute, Buena Park, CA, USA

Shengyu Hua Tianjin University of Traditional Chinese Medicine, Tianjin, China

Chun-Yung Huang Department of Seafood Science, National Kaohsiung Marine University, Kaohsiung, Taiwan

Guangling Jiao Aquatic and Crop Resource Development, National Research Council of Canada, Halifax, NS, Canada; Coastal Zones Research Institute Inc., Shippagan, NB, Canada

Phillip Joy Department of Applied Human Nutrition, Mount Saint Vincent University, Halifax, NS, Canada

Priya Kathirvel Department of Applied Human Nutrition, Mount Saint Vincent University, Halifax, NS, Canada

Chun Li China Academy of Chinese Medical Sciences, Institute of Chinese Materia Medica, Beijing, China

Haixia Li School of Pharmaceutical Sciences, Zhengzhou University, Zhengzhou, China

Minhui Li Baotou Medical College, Baotou, Inner Mongolia, China

Pengfei Li Science and Technology Department, Henan University of Traditional Chinese Medicine, Zhengzhou, Henan, China

Raorao Li Institute of Chinese Materia Medica, China Academy of Chinese Medical Sciences, Beijing, China

Yazhuo Li State Key Laboratory of Drug Delivery Technology and Pharmacokinetics, Tianjin Institute of Pharmaceutical Research, Tianjin, China 
Yingqin Li Nutrilite Health Institute, Buena Park, CA, USA

Bing Lin College of Pharmacy, Guizhou University, Guiyang, Guizhou, China; Guizhou Engineering Center for Innovative Traditional Chinese Medicine and Ethnic Medicine, Guiyang, Guizhou, China

Li-mei Lin School of Pharmacy, Hunan University of Chinese Medicine, Changsha, China

Changxiao Liu State Key Laboratory of Drug Delivery Technology and Pharmacokinetics, Tianjin Institute of Pharmaceutical Research, Tianjin, China

Fan Liu China-ASEAN Traditional Medicine Exchange and Cooperation Center, Guangxi Botanical Garden of Medicinal Plant, Nan'ning, China

Jianhui Liu College of Pharmacy and Bioengineering, Chongqing University of Technology, Chongqing, China; Chongqing Technology and Business University, Chongqing, China

Ta-si Liu Hunan University of Traditional Chinese Medicine, Changsha, China

Tongxiang Liu Institute of Chinese Minority Traditional Medicine, Minzu University of China, Beijing, China

Xiongli Liu College of Pharmacy, Guizhou University, Guiyang, Guizhou, China; Guizhou Engineering Center for Innovative Traditional Chinese Medicine and Ethnic Medicine, Guiyang, Guizhou, China

Yanze Liu Institute of Medicinal Plant Development, Chinese Academy of Medical Sciences and Peking Union Medical College, Beijing, China

Ying-Jiao Liu Hunan University of Traditional Chinese Medicine, Changsha, China

Fei Long School of Pharmacy, Chengdu University of Traditional Chinese Medicine, Chengdu, China

Chunbo Lu School of Pharmaceutical Sciences, Zhengzhou University, Zhengzhou, China

Guanghua Lu School of Pharmacy, Chengdu University of Traditional Chinese Medicine, Chengdu, China

Xuran Lu School of Traditional Chinese Medicine, Capital Medical University, Beijing, China

Yin Lu Department of Pharmacology, Nanjing University of Chinese Medicine, Nanjing, Jiangsu, China

Bohdan L. Luhovyy Department of Applied Human Nutrition, Mount Saint Vincent University, Halifax, NS, Canada 
Mingsan Miao Science and Technology Department, Henan University of Traditional Chinese Medicine, Zhengzhou, Henan, China

Zifan Pei Department of Pharmacology, College of Pharmacy, Nanjing University of Chinese Medicine, Nanjing, Jiangsu, China

Wenhui Qian Department of Pharmacology, College of Pharmacy, Nanjing University of Chinese Medicine, Nanjing, Jiangsu, China

Panbo Qiu Science and Technology Department, Henan University of Traditional Chinese Medicine, Zhengzhou, Henan, China

Caifang Wang Capital Medical University, Beijing, China

Ling Wang College of Pharmacy, Henan University of Traditional Chinese Medicine, Zhengzhou, China

Yulan Wang Dalian Institute for Drug Control, Dalian, China

Zhengtao Wang Shanghai University of Traditional Chinese Medicine, Shanghai, China

Zhimin Wang Institute of Chinese Materia Medica, China Academy of Chinese Medical Sciences, Beijing, China

Zhuju Wang Institute of Chinese Materia Medica, China Academy of Chinese Medical Sciences, Beijing, China

Zongwei Wang Massachusetts General Hospital, Harvard Medical School, Boston, MA, USA

Hua Wei Institute of Chinese Materia Medica, China Academy of Chinese Medical Sciences, Beijing, China; Key Laboratory of Plant Resources Conservation and Utilization, College of Biology and Environmemtal Science, Jishou University, Jishou, China

Yingfang Wei School of Pharmacy, Chengdu University of Traditional Chinese Medicine, Chengdu, China

Jiandong Wu Key Laboratory of Marine Drugs, Ministry of Education, Shandong Provincial Key Laboratory of Glycoscience and Glycotechnology, School of Medicine and Pharmacy, Ocean University of China, Qingdao, Shandong, China

Lihong Wu Shanghai R\&D Centre for Standardization of Chinese Medicines, Shanghai University of Traditional Chinese Medicine, Shanghai, China

Xuelei Xin Xinjiang Technical Institute of Physics and Chemistry, Chinese Academy of Sciences, Urumqi, Xinjiang, China

Bei Xu Hunan University of Traditional Chinese Medicine, Changsha, China

Hong Xu Shanghai University of Traditional Chinese Medicine, Shanghai, China 
Jun Xu School of Chinese Medicine, Hong Kong Baptist University, Hong Kong, China

Jie Yan School of Pharmacy, Chengdu University of Traditional Chinese Medicine, Chengdu, China

Li-hua Yan Institute of Chinese Materia Medica, China Academy of Chinese Medical Sciences, Beijing, China

Jin Yang Beifang University of Nationalities, Ningxia, China

Li Yang Shanghai University of Traditional Chinese Medicine, Shanghai, China

Yang Yi College of Food Science and Engineering, Wuhan Polytechnic University, Wuhan, China

Fei Yin College of Pharmacy and Bioengineering, Chongqing University of Technology, Chongqing, China

Fei Yu Tianjin Key Laboratory on Technologies Enabling Development of Clinical Therapeutics and Diagnostics, School of Pharmacy, Tianjin Medical University, Tianjin, China

Guangli Yu Key Laboratory of Marine Drugs, Ministry of Education, Shandong Provincial Key Laboratory of Glycoscience and Glycotechnology, School of Medicine and Pharmacy, Ocean University of China, Qingdao, Shandong, China

Wen-Jen Yu Department of Biotechnology, Hung Kuang University, Taichung, Taiwan

Feng Zhang Department of Pharmacology, Nanjing University of Chinese Medicine, Nanjing, Jiangsu, China

Jun Zhang Tianjin Key Laboratory on Technologies Enabling Development of Clinical Therapeutics and Diagnostics, School of Pharmacy, Tianjin Medical University, Tianjin, China

Junzeng Zhang Aquatic and Crop Resource Development, National Research Council of Canada, Halifax, Canada

Ming-wei Zhang Sericultural and Agri-Food Research Institute, Guangdong Academy of Agricultural Sciences, Guangzhou, China

Qi-wei Zhang China Academy of Chinese Medical Sciences, Institute of Chinese Materia Medica, Beijing, China

Xiaozhe Zhang Dalian Institute of Chemical Physics, Chinese Academy of Sciences, Dalian, China

Xiao-liang Zhao Experimental Research Center, China Academy of Chinese Medical Sciences, Beijing, China 
Xiaoliang Zhao Key Laboratory of Marine Drugs, Ministry of Education, Shandong Provincial Key Laboratory of Glycoscience and Glycotechnology, School of Medicine and Pharmacy, Ocean University of China, Qingdao, Shandong, China

Yang Zhao Key Laboratory for Information System of Mountainous Areas and Protection of Ecological Environment, Guizhou Normal University, Guiyang, China; The Research Center for Quality Control of Natural Medicine, Guizhou Normal University, Guiyang, China

Yuqing Zhao School of Traditional Chinese Medicine, Shenyang Pharmaceutical University, Shenyang, China

Xidan Zhou Institute of Chinese Materia Medica, China Academy of Chinese Medical Sciences, Beijing, China

Xin Zhou Key Laboratory for Information System of Mountainous Areas and Protection of Ecological Environment, Guizhou Normal University, Guiyang, China; The Research Center for Quality Control of Natural Medicine, Guizhou Normal University, Guiyang, China

Ying Zhou College of Pharmacy, Guizhou University, Guiyang, Guizhou, China; Guizhou Engineering Center for Innovative Traditional Chinese Medicine and Ethnic Medicine, Guiyang, Guizhou, China

En-yuan Zhu Shanghai University of Traditional Chinese Medicine, Shanghai, China

Jing-jing Zhu Institute of Chinese Materia Medica, China Academy of Chinese Medical Sciences, Beijing, China 